\title{
Lama und Alpaka Teil II - Bedeutende Erkrankungen der Neuweltkameliden
}

Sonja Franz

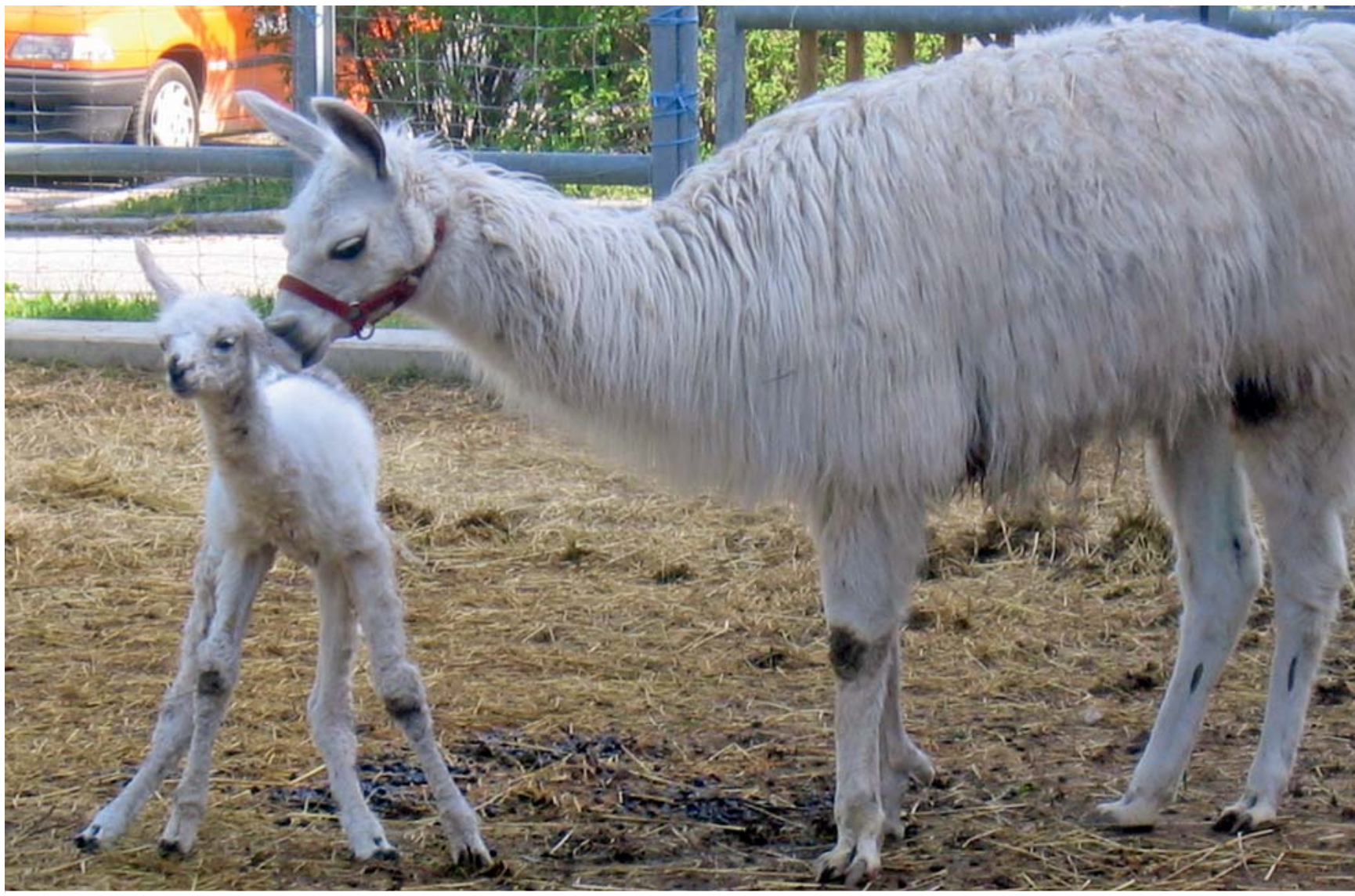

(c) S. Franz

Lesen Sie in Teil II der „Kamelidenserie“, welche bedeutenden Erkrankungen es gibt, welche diagnostischen Verfahren weiterhelfen, und welche Therapien für diese besonderen Tiere passend sind.

Durch die steigende Beliebtheit der Neuweltkamelidenhaltung in vielen europäischen Ländern finden sich diese Tiere natürlich auch vermehrt als Patienten in der Tierarztpraxis wieder. Die vielen Besonderheiten dieser Tiere, die in ihrem Wesen bzw. Verhalten und in ihren morphologisch-physiologischen Merkmalen liegen, erschweren oftmals die diagnostische und auch therapeutische Herangehensweise und erkrankte Lamas und Alpakas stellen somit für den behandelnden Tierarzt eine wahre Herausforderung dar. Als sogenannte „Fluchttiere“ zeigen Neuweltkameliden zudem im Krankheitsfall klinische Symptome sehr spät, d. h. oftmals erst dann, wenn eine Erkran- kung schon weit fortgeschritten ist. Dies macht eine erfolgreiche Behandlung in vielen Fällen schwierig, womit der Früherkennung von Krankheitssymptomen eine groBe Bedeutung zukommt.

\section{Diagnostische Möglichkeiten}

Erkrankungen, die bei Neuweltkameliden auftreten, sind im Allgemeinen auch bei anderen Tierspezies bekannt. Doch einige Krankheitsbilder ergeben sich aus den Besonderheiten dieser Tiere, weshalb Wissen über Anatomie und Physiologie dieser Tiere grundsätzlich erforder- 
lich ist (s. Teil I der „Kamelidenserie“ im Veterinär Spiegel Ausgabe Nr. 4, 2017).

Zur Diagnostik stehen neben der Erhebung einer exakten Anamnese und der klinischen Untersuchung auch Laboruntersuchungen wie z.B. Blut, Kot, Liquor, Peritonealflüssigkeit, Futtermittelanalysen, etc. zur Verfügung. Die Anwendung von bildgebenden Verfahren in den unterschiedlichsten Krankheitsfällen, wie die Röntgenuntersuchung, die Sonografie, die Computertomographie oder die Magnetresonanztomographie, eignet sich bei diesen Tieren hervorragend für diagnostische Zwecke. Diese Verfahren geben oftmals hilfreiche Hinweise zur Abklärung der Krankheitsursache.

\section{Gastrointestinaltrakt}

Erkrankungen des Gastrointestinaltraktes gehören zu den am häufigsten auftretenden Erkrankungen bei Neuweltkameliden. Hierzu zählen vor allem Enteritiden, die Kompartimentazidose sowie die Entstehung von Magenoder Darmgeschwüren $[3,5,10]$. Die Ursachen einer Enteritis können infektiöser oder nicht infektiöser Natur sein. Zu den infektiösen Ursachen zählen vor allem Durchfallerkrankungen von Neugeborenen, hervorgerufen durch Rotaviren, Coronaviren oder E. coli. Bei älteren, adulten Tieren stehen Clostridieninfektionen ( $\mathrm{Cl}$. perfringens) im Vordergrund, wobei den Typen A, C und D die größte Bedeutung zugeschrieben wird.

\section{Endoparasiten}

Endoparasitosen zählen zu den häufigsten infektiösen Ursachen von Enteritiden bei Neuweltkameliden, sowohl bei Jungtieren, als auch bei adulten Tieren. Die Besonderheit bei Neuweltkameliden im Gegensatz zu anderen Tierarten liegt darin, dass bei einer vorhandenen Endoparasitose in den meisten Fällen kein Durchfall als klinisches Symptom auftritt. Im akuten Krankheitsfall sind die Symptome eher unspezifisch und reichen von verminderter oder keiner Fresslust, über Kolik bis hin zum Festliegen. Bei längerem Fortbestehen der Erkrankung ist eine Abmagerung des Tieres als sehr typisch zu beobachten ( $\bullet$ Abb. 1). Die häufig nachgewiesenen Endoparasiten bei Neuweltkameliden umfassen Protozoen, Nematoden, Zestoden und Trematoden [2].

\footnotetext{
Merke

Als Besonderheit, im Vergleich zu anderen Tierarten, gilt die Infektion von Neuweltkameliden mit dem Kleinen Leberegel (Dicrocoelium dendriticum), die im Gegensatz zu Rindern und kleinen Wiederkäuern bei Lamas und Alpakas zu einem schwereren Krankheitsverlauf führt. Dieses Krankheitsbild (Dicrocoeliose) ist hauptsächlich aus dem europäischen Raum bekannt. Die Leber zeigt dabei massive Veränderungen hinsichtlich der Entstehung von Leberabszessen, -granulomen und einer Leberzirrhose. Bleibt die
}

Dicrocoeliose unbehandelt, so führt diese oftmals zum Tod des Tieres [12, 13, 21].

Zu den Antiparasitika, die für die Therapie eines Dicrocoelium dendriticum Befalles bei Neuweltkameliden eingesetzt werden, zählen Albendazol und Praziquantel. Bei der Verabreichung von Albendazol gibt es Hinweise in der Literatur, dass bei Neuweltkameliden eine toxische Wirkung auftreten kann. Todesfälle bei Alpakas im Zusammenhang mit der Gabe von Albendazol wurden bereits beschrieben. Neben der toxischen Wirkung weist Albendazol auch nur eine Teilwirkung gegenüber diesem Parasiten auf. Als Mittel der Wahl gilt demnach der Wirkstoff Praziquantel, der zur Bekämpfung des Kleinen Leberegels in einer Dosierung von $50 \mathrm{mg} / \mathrm{kg}$ oral verabreicht werden muss $[4,7]$.

\section{Kokzidien}

Bei Neuweltkameliden sind bislang 6 Kokzidienarten (wirtsspezifische Eimerienarten) beschrieben: E. lamae, E. punoensis, E. ivitaensis, E. macusaniensis, E. alpacae, E. peruviana. Während bei anderen Tierarten ein Kokzidienbefall eher als Jungtiererkrankung angesehen wird, ist bei den Neuweltkameliden diese Infektion durchaus bei adulten Tieren, oftmals auch mit Todesfolge zu beobachten. Zur Therapie werden Aryltriazine (Toltrazuril und Diclazuril) oral verabreicht. Toltrazuril wird derzeit in einer Dosierung von 20 mg/kg p. o. empfohlen [7].

\begin{abstract}
Merke
Generell ist wichtig zu erwähnen, dass eine ausschließliche medikamentöse Behandlung für die Parasitenbekämpfung natürlich auch bei Neuweltkameliden zur Sanierung eines Bestandes nicht ausreicht. Neben der Gabe von Anthelmintika ist die Optimierung des Hygiene- und Weidemanagements auch bei diesen Tieren ein wesentlicher Bestandteil eines erfolgreichen Parasitenbekämpfungsprogrammes.
\end{abstract}

\section{Kompartmentazidose}

Als nicht infektiöse Ursache von Enteritiden gelten auch fütterungsbedingte Ursachen. In diesem Zusammenhang ist die C1-Azidose zu nennen (Neuweltkameliden haben ein Magensystem mit 3 Kompartimenten: C1-C3). Die Kompartimentazidose tritt bei Neuweltkameliden aus denselben Gründen wie beim Wiederkäuer auf, d. h. die Aufnahme von übermäßigen Mengen an Kraftfutter oder zucker- bzw. stärkehaltigen Futtermitteln führt in der Regel zu einer Laktatazidose mit pH-Werten des Mageninhaltes (C1) von $<5,0$. In diesem Zusammenhang ist ein plötzlicher Futterwechsel oftmals als krankheitsauslösend anzusehen. In Abhängigkeit der aufgenommenen Futtermenge und Dauer der Erkrankung zeigen diese Tiere Fressunlust, breiigen Kot und Koliksymptome. Es kommt zum Festliegen und in vielen Fällen auch zum Tod des Tieres. 


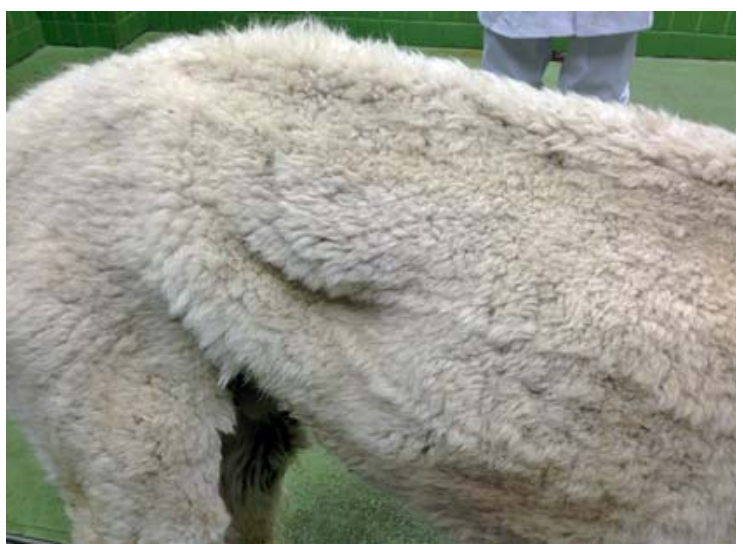

- Abb. 1 Hochgradige Abmagerung eines Alpakas. Der Ernährungszustand wird stets durch Palpation der Bemuskelung im Bereich der Lendenwirbelsäule palpiert. Bei diesem Alpaka ist eine deutliche Abmagerung bereits mit bloßem Auge erkennbar. ( S. Franz

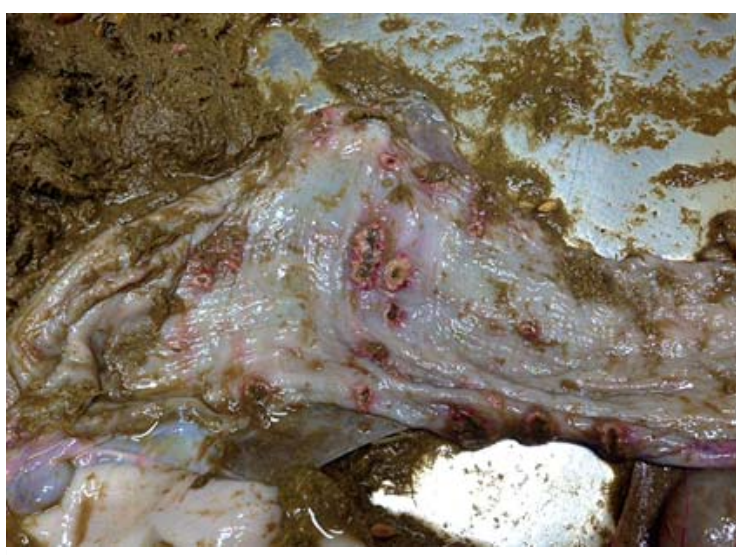

- Abb. 2 Magengeschwüre im Bereich des dritten Magenkompartiments (C3). @ S. Franz

mens kann in diesem Fall wertvolle diagnostische Hinweise liefern.

\section{Merke}

Da Stress als Hauptursache für die Entstehung von Magenulzera gesehen wird, ist es für eine erfolgreiche Therapie von größter Bedeutung, die Stressquelle zu identifizieren. Dies stellt sich oftmals als nicht einfach heraus, da gewisse Situationen für Tiere einen Stress darstellen können, die als solche für Tierhalter nicht erkannt werden.

Die Therapie von Ulzera erweist sich als schwierig. Neben einer symptomatischen Therapie (kein Kraftfutter, Füttern von Heu) sind Magenulzera mit dem Wirkstoff Omeprazol (Protonenkanalblocker) in einer Dosierung von $1 \mathrm{mg} / \mathrm{kg}$ i.v. zu behandeln. Omeprazol kann auch oral in einer Dosierung von $2 \mathrm{mg} / \mathrm{kg}$ zur Prophylaxe und $4 \mathrm{mg} /$ $\mathrm{kg}$ zur Therapie eingesetzt werden. Die Effektivität einer oralen Behandlung ist jedoch nicht vergleichbar mit einer intravenösen Gabe, da bei dieser Applikationsform eine sehr schlechte Bioverfügbarkeit gegeben ist.

\section{Haut}

Literaturberichten zu Folge zählen Hauterkrankungen neben Endoparasitosen zu den am häufigsten auftretenden Krankheitsbildern bei Neuweltkameliden. Beschriebene Ursachen sind infektiöser Natur (Bakterien, Pilze, Ektoparasiten) und nichtinfektiöser Natur. Dazu zählen Autoimmunerkrankungen (z. B. Pemphigus vulgaris), Mangelerkrankungen (z.B. Zinkmangel), Neoplasien und Allergien. Als diagnostische Massnahmen bei Hautveränderungen stehen eine exakte Erhebung der Anamnese, die klinische Untersuchung, die Blutuntersuchung, Futtermittelanalyse sowie die Durchführung eines Hautgeschabsels oder einer Hautbiopsie zur Verfügung [3, 5, $6,9,10,17,18]$. 


\section{Zinkmangel}

Ein bestehender Zinkmangel kann zu dem Krankheitsbild „zinc responsive dermatosis“ mit Haarverlust, Hyperkeratose und hochgradig borkiger, verdickter Haut führen, wobei die betroffenen Stellen sehr häufig generalisiert verteilt über den gesamten Körper zu finden sind ( $\bullet$ Abb. 3). Komplikationen bei der zinc responsive dermatosis können vor allem bei Auftreten von Sekundärinfektionen auftreten. Sinnvoll ist, bei Auftreten der genannten Symptomatik, den Zinkgehalt im Blut zu messen und bei einem verminderten Zinkgehalt die Ursache abzuklären. Der physiologische Zinkgehalt im Blut wird mit 10$20 \mu \mathrm{mol} / \mathrm{L}$ angegeben (unterschiedliche Referenzwerte).

Ursächlich werden ein fütterungsbedingter und ein angeborener Zinkmangel angenommen. Bei letzterer Ursache wird eine hereditäre Störung der enteralen Zinkresorption diskutiert. Auch, wenn die Darmschleimhaut durch andere Erkrankungen geschädigt ist (z. B. bei einer Endoparasitose), kann es zu einer Beeinträchtigung der Zinkresorption über den Darm und in Folge zu einem Zinkmangel kommen. Zur Ursachenfindung ist wichtig zu wissen, dass Ca, Fe und Cu als sogenannte Gegenspieler zu Zink fungieren. Befinden sich diese Mengen- bzw. Spurenelemente vermehrt im Organismus, können sie zu einer negativen Beeinflussung der Zinkresorption führen. Demnach sollte der Gehalt der genannten Mengenbzw. Spurenelementen stets zusätzlich kontrolliert werden, um hier entsprechend einwirken zu können.

Therapeutisch erfolgt bei Zinkmangel eine orale Zinksubstitution von Zinksulfat in der Dosierung von $2 \mathrm{~g} / \mathrm{d} /$ Tier oder von Zinkmethionin in einer Dosierung von $4 \mathrm{~g} /$ $\mathrm{d} /$ Tier. Diese Behandlung muss über mehrere Wochen erfolgen, bis sich ein Therapieerfolg einstellt. Nichtsdestotrotz, werden immer wieder Patienten vorgestellt, bei denen ein Zinkmangel unklarer Genese besteht und auch therapeutisch nicht in den Griff zu bekommen ist. Demnach ist die genaue Ursache für das Entstehen dieser Erkrankung ist bei Neuweltkameliden sicherlich noch weiter zu erforschen.

\footnotetext{
Merke

Trockene, schuppige und hyperkeratotische Haut kann sowohl durch Zinkmangel (angeboren oder fütterungsbedingt) als auch durch Ektoparasiten wie Sarkoptes-, Chorioptes-, oder Psoroptesmilben hervorgerufen werden. Eine sorgfältige diagnostische Aufarbeitung ist hier Pflicht, um die korrekte Therapie einzuleiten. Zudem hat Sarkoptes bei Lama und Alpaka zoonotisches Potenzial!
}

\section{Milbenbefall}

Ein Milbenbefall kann bei Neuweltkameliden (ebenso wie Zinkmangel) das Krankheitsbild der Hyperkeratose ausbilden. Hier steht vor allem der Befall mit Sarcoptes spp. und Chorioptes spp. im Vordergrund. Sarcoptes scabiei

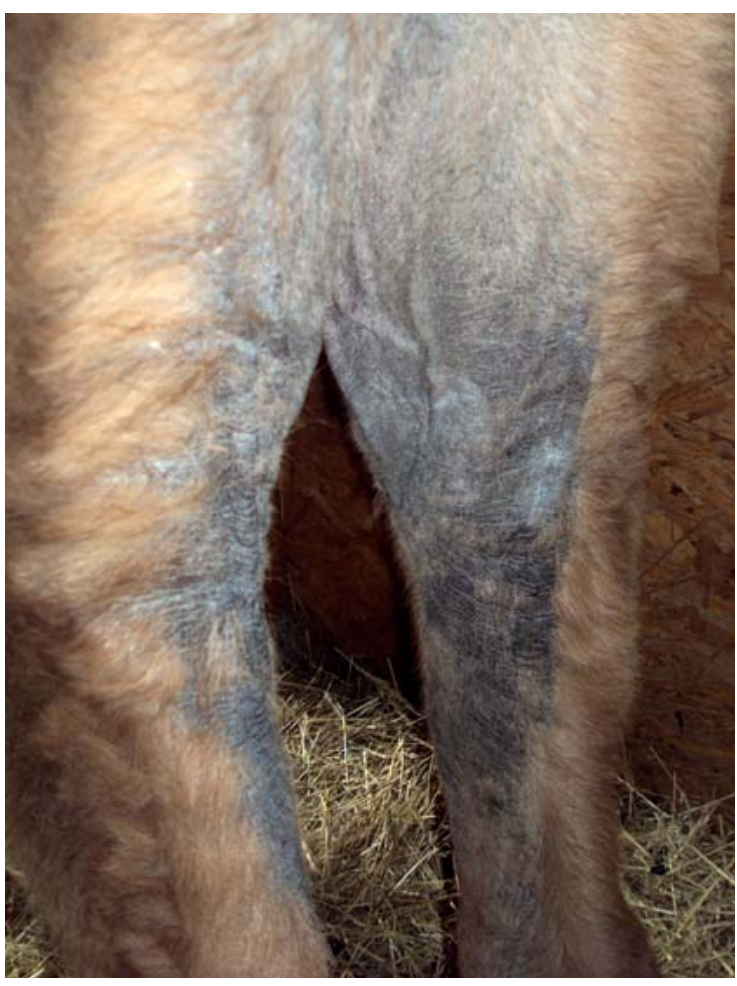

- Abb. 3 Generalisierte Hyperkeratose im Bereich der Hinterextremitäten im Rahmen eines Zinkmangels. (c) S. Franz

var. auchinae ist besonders zu erwähnen, da er auch auf den Menschen übergehen kann und somit ein Zoonosepotenzial besitzt. Sarkoptesmilben befinden sich vorrangig im Zwischenzehenbereich, im Bereich der Innenschenkel und des ventralen Abdomens. Psoroptesmilben sind vermehrt im Ohrbereich anzutreffen und rufen dann eine Ohrenentzündung (Otitis externa) hervor. Eine Ausbreitung dieser Milben auf den Rumpf ist ebenso möglich. Chorioptes spp. befallen vor allem Schwanz, Anus und den Bereich der Schenkelinnenseiten und den Zwischenzehenbereich ( $\bullet$ Abb. 4).

Der Milbennachweis erfolgt mikroskopisch nach Durchführung eines tiefen Hautgeschabsels im Randbereich der Hautveränderung, wobei ein negativer Befund nicht beweisend ist und die Diagnostik erschwert. Therapeutisch sind Wirkstoffe (als Injektion oder topisch) einzusetzen, die zu den makrozyklischen Laktonen zählen, wobei in der Literatur ein wirkungsvolles Therapieschema noch kontrovers diskutiert wird, es gibt bis dato diesbezüglich keine wissenschaftlich fundierten Studien. In jedem Fall ist es wichtig, wenn möglich, die Milbenart vor Therapie zu identifizieren, denn die Therapie gestaltet sich unterschiedlich: Leben Chorioptes spp. vermehrt von Hautschuppen, so ernähren sich Sarcoptes spp. und Psoroptes spp. jeweils von Blut und Lymphe. Eine Therapie mittels „pour on“ Präparaten ist deshalb bei der Milbenart vor- 


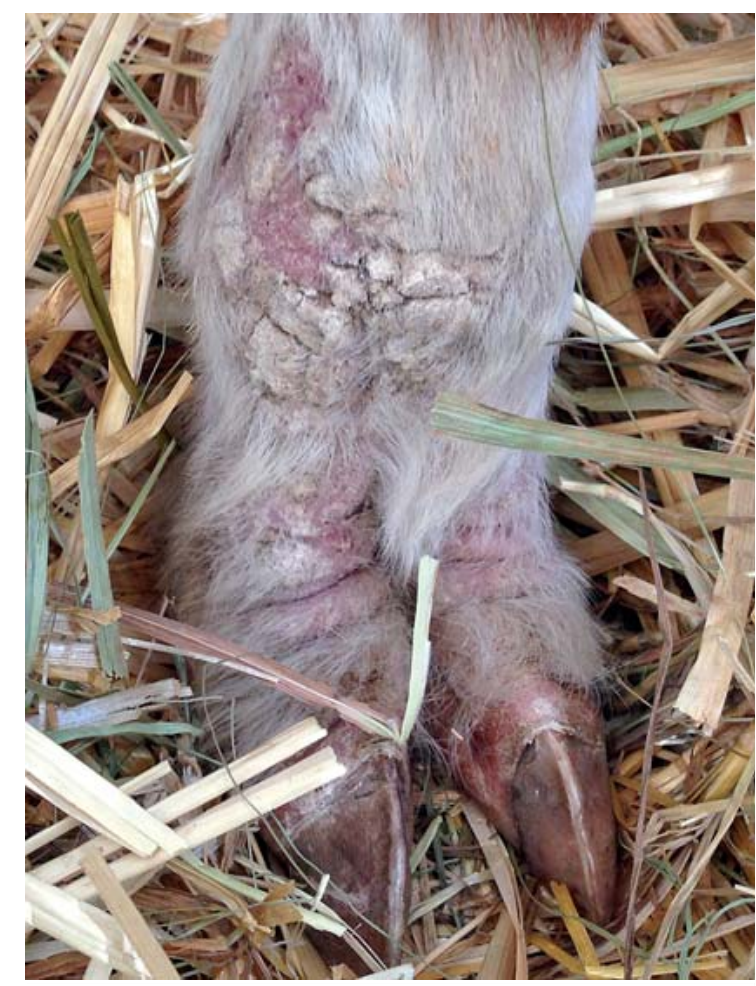

- Abb. 4 Hyperkeratotische Hautveränderung im Zusammenhang mit einer Chorioptesmilbeninfektion im Bereich der distalen Extremitäten. () S. Franz

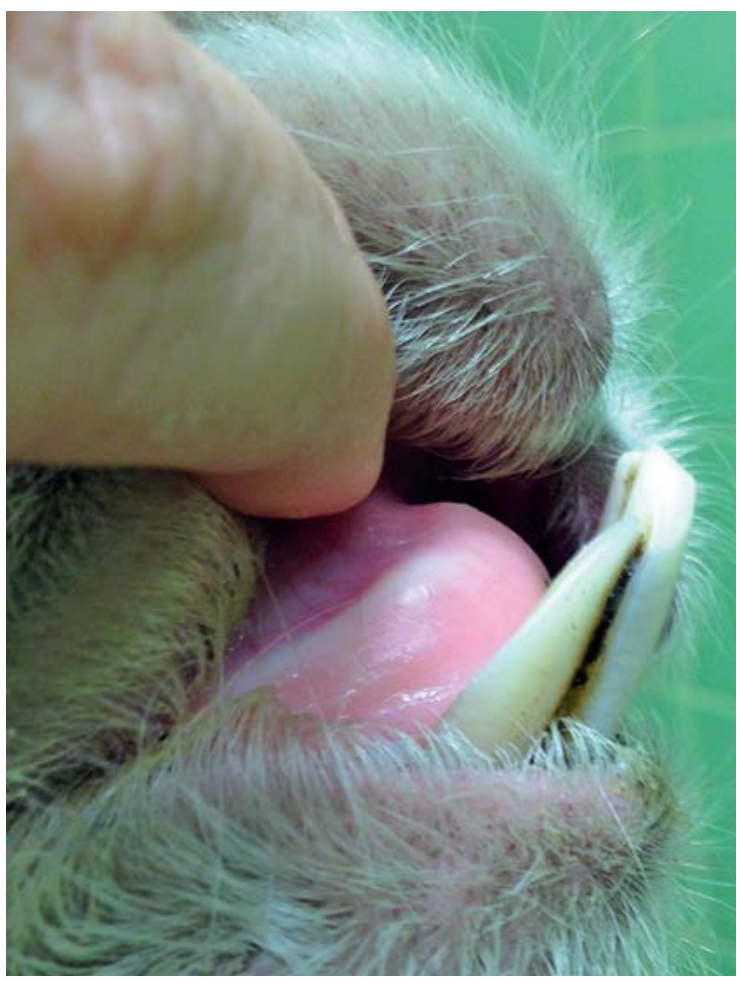

- Abb. 5 Überlange Schneidezähne des Unterkiefers. Physiologischerweise sollten die Inzisivi des Unterkiefers mit der Kauplatte des Oberkiefers abschließen. @ S. Franz

Abmagerung und Wickelkauen sind weitere klinische Symptome, die sich bei längerem Bestehen dieses Krankheitsbildes entwickeln. Eine endgültige Diagnose kann nur anhand von Röntgenbildern des betroffenen Kiefers gestellt werden und ist bei diesen Patienten in jedem Fall vorzunehmen, auch in Hinblick darauf, ob eine Osteomyelitis bereits vorliegt oder nicht. Wird eine Zahnwurzelinfektion diagnostiziert, so wird in der Regel eine Extraktion des betroffenen Zahnes empfohlen.

Als weitere Zahnerkrankungen sind auch die bei Neuweltkameliden häufig vorkommenden angeborenen Kiefermissbildungen mit entweder zu kurzem Oberkiefer oder zu langem Unterkiefer, zu nennen. Die Folgen liegen im unterschiedlichen Abrieb der Zähne und den damit einhergehenden Verletzungen an der Schleimhaut, die wiederrum zu entzündlichen Veränderungen des Zahnhalteapparates führen können.

\section{Mykoplasmeninfektion}

Eine Infektion mit dem zellwandfreien Bakterium Candidatus Mycoplasma haemolamae (CMhl), das an der Oberfläche von Erythrozyten parasitiert [14], kann bei betroffenen Neuweltkameliden zu einer akuten Erkrankung mit einer hämolytischen Anämie, Ödembildung, Schwäche, bis hin zum Festliegen und zum Tode führen 


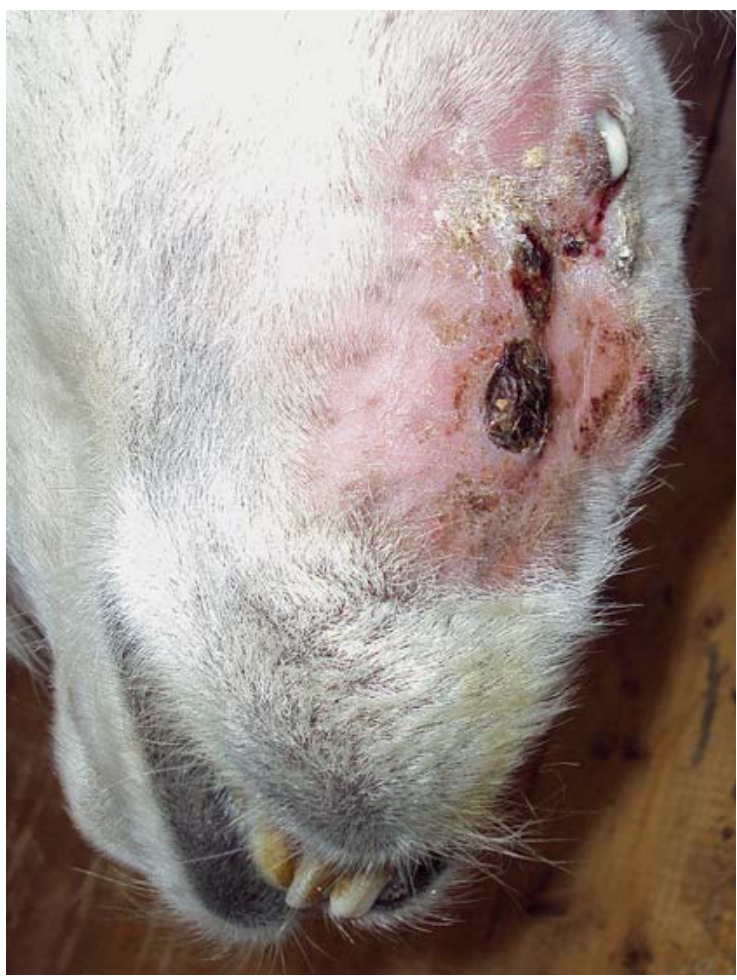

- Abb. 6 Alpaka mit Zahnwurzelabszessen im Bereich des Unterkiefers. @ S. Franz

( $\bullet$ Abb. 7). In den meisten Fällen führt eine Infektion bei Neuweltkameliden aber zu einem chronischen Trägertum, ohne dass es zum Ausbruch klinischer Symptome kommt [8]. Bei solchen Tieren liegen aber auch Berichte über das Auftreten von Abmagerung, Schwäche oder Fruchtbarkeitsstörungen vor. Diese Trägertiere sind aber dahingehend gefährdet, dass Stresssituationen unterschiedlicher Art, die auf das Tier einwirken, eine akute Erkrankung mit oben beschriebener Symptomatik auslösen können. Zu Stresssituationen zählen, Transport, Teilnahme an Shows, Rangkämpfe in der Herde, Platzmangel, Deckzeitpunkt, Geburt, aber auch das gleichzeitige Bestehen anderer Erkrankungen, wie z. B. einer Endoparasitose.

Der Übertragungsweg der Haemoplasmen ist bis dato noch nicht vollständig geklärt. Neben der direkten Übertragung von Tier zu Tier über das Blut gelten blutsaugende Insekten als wahrscheinlichste Infektionsquelle. Auch eine vertikale Übertragung des Erregers scheint möglich und wird diskutiert [20]. Die Diagnose erfolgt durch eine Blutuntersuchung (EDTA-Blut) mittels PCR-Technik [19] und eine Therapie ist nur sinnvoll bei akut erkrankten Tieren. Dabei spielt die Durchführung einer Bluttransfusion eine wesentliche Rolle. Die zusätzliche Gabe von Tetrazyklinen über einen längeren Zeitraum $(20 \mathrm{mg} / \mathrm{kg}$ i.m. über mindestens 10 Tage) wird empfohlen. Jedoch ist da-

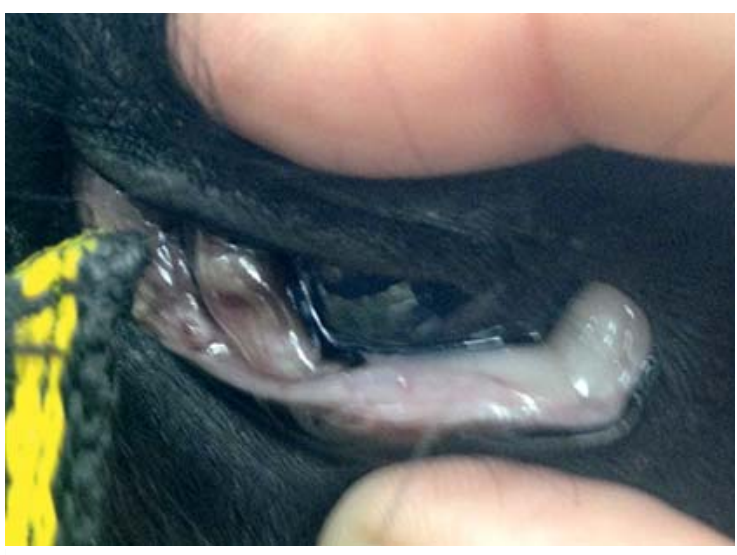

- Abb. 7 Anämische Lidbindehaut eines Alpakas bedingt durch eine akute akute Cand. Mycoplasma haemolamae Infektion. @ S. Franz

rauf hinzuweisen, dass es bis dato noch nicht gelungen ist, den Erreger durch eine antibiotische Therapie vollständig zu eliminieren.

\section{Korrespondenzadresse}

A. Univ. Prof. Dr. Sonja Franz
Klinik für Wiederkäuer
Department für Nutztiere und Öffentliches Gesundheitswesen
in der Veterinärmedizin
Veterinärmedizinische Universität Wien (Vetmeduni Vienna)
Veterinärplatz 1
1210 Wien
Österreich

Literatur

[1] Anderson DE. Periapical tooth root infections in llamas and alpacas. Small Rum Res 2006; 61: 235-24

[2] Ballweber RL. Ecto- und Endoparasites of New World Camelids. Vet Clin North Am Food Anim Pract 2006; 25: 295-310

[3] Cebra CK, Anderson D, Tibary A, Van Saun R, Johnson Larue W. Llama and Alpaca Care. Medicine, Surgery, Reproduction, Nutrition, and Health Care. 1st edition. St. Louis, MO, USA: Elsevier; 2014

[4] Dadak AM, Wieser C, Joachim A, Franz S. Efficacy and safety of oral praziquantel against Dicrocoelium dendriticum in llamas. Vet Parasitol 2013; 197: 122-125

[5] Fowler ME. Medicine and Surgery of South American Camelids-Llama, Alpaca, Vicuña, Guanaco. 2nd edition. Ames, lowa: lowa State University Press; 1998

[6] Foster A, Jackson A, Dalterio GL. Skin diseases in South American Camelids. In Pract 2007; 29: 216-223

[7] Franz S, Wittek T, Joachim A, Hinney B, Dadak AM. Llamas and alpacas in Europe: Endoparasites of the digestive tract and their pharmacotherapeutic control. Vet J 2015; 204: 255-262

[8] Franz S, Spergser J, Schwendenwein I, Stanitznig A, Lambacher B, Tichy A, Wittek T. Zum Vorkommen von „Candidatus Mycoplasma haemolamae“ bei klinisch unauffälligen Neuwelt- 
kameliden in Österreich. Berl Munch Tierarztl 2016; 129: 318 322

[9] Franz S, Dadak AM. Hauterkrankungen bei Neuweltkameliden. News4vets 2017; 3: 8-12

[10] Gauly M, Vaughan J, Cebra C. Neuweltkameliden-Haltung, Zucht, Erkrankungen. 3. Aufl., Stuttgart: Enke; 2011

[11] Geurden T, Deprez P, Vecruysse J. Treatment of sarcoptic, psoroptic, and chorioptic manage in a Belgian alpaca herd. Vet Rec 2003; 153: 331-332

[12] Gunsser I, Hänichen T, Maierl J. Leberegelbefall bei Neuweltkameliden. Tierärztl Prax 1999; 27 (G): 187-192

[13] Klein D, Prosl H, Thaller D, Flöck M. Diagnosis of a Dicrocoelium dendriticum infection in New World camelids: a case report. Veterinarni Medicina 2012; 57: 154-162

[14] Messik JB. Haemotrophic mycoplasmas (haemoplasmas): a review and new insights into pathogenic potential. Vet Clin Pathol 2004; 33: 2-13

[15] Niehaus AJ. Tooth root abscesses in llamas and alpacas, 123 cases (1994-2005). J Am Vet Med Assoc 2009; 231: 284-289

[16] Niehaus AJ. Dental Disease in Llamas and Alpacas. Vet Clin Food Anim Pract 2009; 25: 281-293
[17] Royschuk RAW. Llama dermatology (update). Vet Clin North Am Food Anim Pract 1994; 10: 228-239

[18] Scott DW, Vogel JW, Fleis RI, Miller Jr WH, Smith MC. Skin diseases in the alpaca (Vicugna pacos): a literature review and retrospective analysis of 68 cases (Cornell University 19972006). Vet Dermatol 2011; 22: 2-16

[19] Tornquist SJ, Boeder LJ, Cebra CK, Messick J. Use of a polymerase chain reaction assay to study response to oxytetracycline treatment in experimental Candidatus Mycoplasma haemolamae infection in alpacas. Am J Vet Res 2009; 70: 1102-1107

[20] Tornquist S], Boeder LJ, Lubbers S, Cebra CK. Investigation of Mycoplasma haemolamae infection in crias born to infected dams. Vet Rec 2011; 168: 380a

[21] Wenker C, Hatt JM, Hertzberg H, Ossent P, Hänichen T, Brack A, Isenbügel E. Dikrozöliose bei Neuweltkameliden. Tierärztl Prax 1998; 26 (G): 355-361

Bibliografie

DOI https://doi.org/10.1055/s-0043-118896

veterinär SPIEGEL 2018; 28: 15-21

(c) Sonntag Verlag in Georg Thieme Verlag KG Stuttgart .

New York ISSN 0940-8711 